\title{
Histopathologic correlates of clinically verrucous lesions
}

\author{
Leena Dennis Joseph ${ }^{1}$, C. N. Sai Shalini ${ }^{2, *}$, Subalakshmi B. ${ }^{3}$, Divya D. ${ }^{4}$, V. Pavithra ${ }^{5}$ \\ ${ }^{\mathbf{1}}$ Professor, ${ }^{\mathbf{2}}$ Associate Professor, ${ }^{\mathbf{3}, \mathbf{4}}$ Demonstrator, ${ }^{\mathbf{5}}$ Assistant Professor, Dept. of Pathology, Sri Ramachandra \\ Medical College and Research Institute. Sri Ramachandra University, Chennai
}

*Corresponding Author:

Email: saishalini_cn14@yahoo.com

\begin{abstract}
Introduction: Verrucous lesions present as cauliflower like, slowly growing masses, which may be single, multiple or diffuse involving the broad areas of skin or mucosal surfaces. Clinically these lesions are diagnostic challenges. Histopathology remains the gold standard in diagnosing this diverse group of verrucous lesions. The aim of this study was to understand the microscopy of clinically verrucous lesions.

Material and Methods: This is a retrospective data of clinically verrucous lesions over the past five years. Clinical details were obtained from the archives and diagnosis was noted from the pathology records. A total of 116 clinically verrucous lesions were included.

Results: Majority of the cases were Verruca vulgaris (37 cases), followed by 33 cases of Seborrheic keratosis. There were 12 cases of verrucous carcinoma, seven cases of tuberculosis verrucosa cutis, six cases of verrucous nevus and one case of verrucous hemangioma. The other cases were of varied histology, inspite of clinically verrucous appearance. All the cases were diagnosed purely based on histopathology findings, which show characteristic morphological features, which help the pathologist in the correct diagnosis.

Conclusions: Histopathologic confirmation helps in alleviating the anxiety of the patients and helps us to arrive at a correct diagnosis.
\end{abstract}

Keywords: Carcinoma, Papillomatosis, Seborrheic keratosis, Verrucous, Wart.

Received: $03^{\text {rd }}$ July, 2017

\section{Introduction}

'Verrucose' refers to warty lesions or vegetations on the surface of the skin. Clinically verrucous lesions could be any of the long list of lesions, with a warty surface which includes verruca vulgaris, verrucous carcinoma, seborrheic keratosis, Tuberculosis Verrucosa cutis, verrucous lupus erythematosus, proliferative verrucous leukoplakia. Verrucous nevus or rare entities like verrucous perforating collagenoma, verrucous cyst or a verrucous vascular malformation. Verrucous papillary lesions of the oral cavity are also diagnostic dilemmas, as it involves a broad spectrum of lesions ranging from benign to potentially malignant to frank malignant lesions. Benign spectrum includes squamous papilloma, verruca vulgaris, focal epithelial hyperplasia and condylomas. Verrucous hyperplasias are potentially malignant lesions, which can be differentiated from verrucous carcinoma by the exophytic and endophytic nature of the latter as compared to the pure exophytic lesion of the former.

Location can be varied,but the lesion is quite worrisome to the patient, both cosmetically as well as prognostically. Hence a complete excision with a histopathological confirmation is
Accepted: $19^{\text {th }}$ August, 2017

of paramount significance to assist the clinician in initiating appropriate therapy as well as to alleviate the anxiety of the patient.

The aim of our study was to study the histopathology of all the clinically verrucous lesions and to categorise them accordingly into relevant groups, so as to understand them better.

\section{Material and methods}

This is a retrospective data of all the lesions with a clinically verrucous surface and which was sent for histopathological evaluation. The clinical data was obtained from the requisition slips that accompanied the biopsy specimens. The histopathology diagnosis was obtained from the archives in the department of Pathology. 


\section{Results}

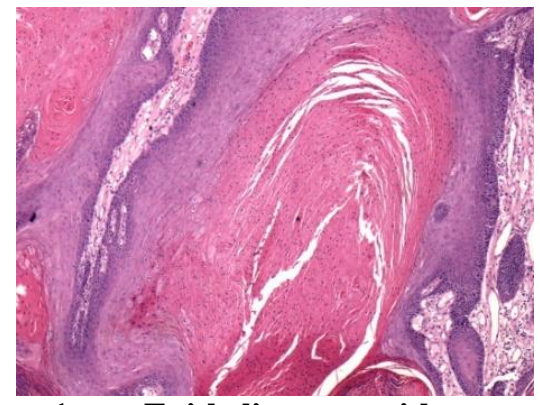

Fig. 1: Epithelium with marked hyperkeratosis and parakeratosis with adjacent epithelium showing koilocytosis (H\&Ex200)

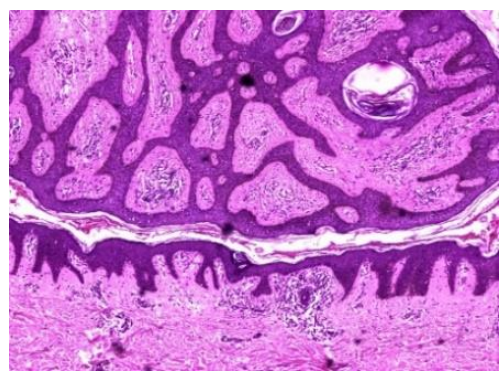

Fig. 2: Epithelium with acanthosis, horn cysts and a classic stuck on appearance (H\&Ex100)

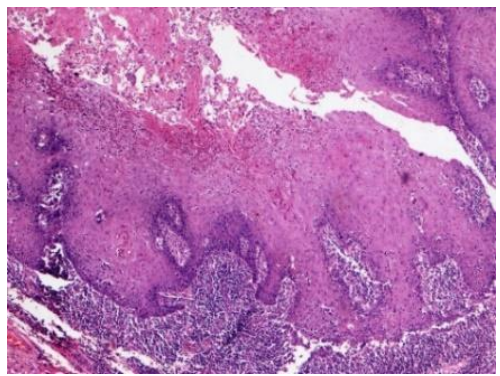

Fig 3: Acanthotic epidermis with broad bulbous rete pegs with dense inflammation (H\&Ex100)

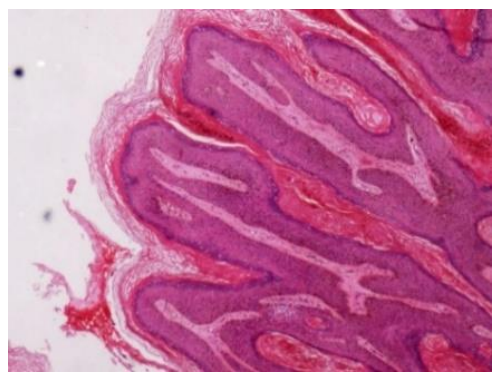

Fig. 4: Epithelium with papillomatosis and hyperkeratosis (H\&Ex100)

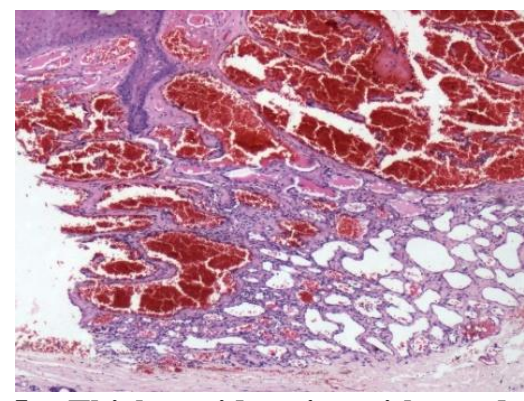

Fig. 5: Thick epidermis with underlying proliferation of capillaries (H\&Ex100)

A total of 116 cases were included in the study. Majority of them were verruca vulgaris, (31.8\%) (Fig. 1) followed closely by seborrheic keratosis (28.4\%) (Fig. 2). There were 12 cases $(10.3 \%)$ of verrucous carcinoma (Fig. 3), 7 cases (6\%) of tuberculosis verrucosa cutis, 6 cases (5.17\%) of verrucous nevus (Fig. 4) and one case of a verrucous hemangioma (Fig. 5). The rest of the cases showed a varied non specific histopathological spectrum with an epidermal acanthosis, papillomatosis and sometimes a psoriasiform hyperplasia, which clinically had a verrucous appearance.

\section{Discussion}

It was in 1941, when Freidell and Rosenthal reported the first oral cavity lesion as papillary verrucoid carcinoma, which was a well differentiated squamous cell carcinoma with a verrucoid appearance.

The clinically polypoid or verrucous lesion needs a histopathological confirmation, at times requiring other ancillary studies like immunohistochemistry or molecular studies. Most of our cases were verruca vulgaris and other related lesions like myrmecia, acrokeratosis verruciformis of Hopf and epidermodysplasia verruciformis. Patients and clinicians experience difficulty in treating these lesions which range from common wart, myrmecia, plantar warts, coalesced mosaic warts. Filiform warts, periungual warts, anogenital warts and respiratory papillomas. A verruca vulgaris or condyloma is an epithelial hyperplasia, induced by Human Papilloma Virus (HPV), mostly the low risk types, 6 and 11. Morphologically the lesion presents with marked papillomatosis, hyperkeratosis and parakeratosis. The rete ridges are elongated and are bent inward so that they appear to point radially toward the center. However the histopathological diagnostic clue is koilocytosis. Other than this vertical tiers of parakeratoticcells and foci of clumped keratohyaline granules are characteristic. 
Virologic tests using in situ hybridisation or Polymerase chain reaction are used to subclassify the virus. Many creams, gels and plasters with salicylic acid is considered therapeutic for viral warts. Cryotherapy or freezing is yet another modality of treatment using liquid nitrogen.

Another very similar polypoid lesion with a cauliflower like appearance is seborrheic keratosis. These lesions are very common, sometimes single, however mostly they are multiple. Their occurrence is usually over the trunk and face. This benign lesion is sharply demarcated, brownish in color and slightly raised with a classic stuck on appearance, if the lesion is excised in toto. Majority of them have a verrucous surface with a soft friable consistency. Some of them are smooth but with keratotic plugs. Some lesions may even be pedunculated, usually over the chest and neck. The microscopy is marked by a exophytic papillary proliferation, hyperparakeratinisation and deep keratin filled invaginations. Squamous and basaloid cells are seen in the acanthotic epidermis. They may present as several variants like acanthotic, reticulated, irritated, hyperkeratotic, clonal and pigmented type. ${ }^{1}$

Both seborrheic keratosis and verruca vulgaris can greatly resemble each other. Yu Hung $\mathrm{Wu}$ et al studied the histopathologic and immune histochemical distinction of the condyloma and seborrheic keratosis in the genitofemoral areas. ${ }^{2}$ Seborrheic keratosis involves the proliferation of both the squamous and basaloid cells, with presence of horn cysts and pseudohorn cysts. The classic stuck on appearance helps in the recognition and quick identification of these lesions. Ancillary techniques Immunohistochemistry with P16 can be used to differentiate the diagnostic ally difficult cases as HPV infected lesions will be positive for P16.

Condylomas, according to Yu Hung Wu had broader, reticulated acanthosis with a fascicular arrangement of keratinocytes, with the clear cut evidence of koilocytes. All our cases of verruca vulgaris and related lesions had the classic features on histopathology.

Another lesion with a classic verrucous appearance is verrucous carcinoma, which is an uncommon variant of squamous cell carcinoma. This malignancy has a close association with tobacco chewing or inhalation of snuff, leading to the cauliflower like growth in the oral cavity. ${ }^{3}$ This occurs most commonly in the oral cavity in the age range of 50 to 70 years and mostly in male patients. Clinically the lesion is a slow growing diffuse exophytic lesion usually covered by leukoplakic patches. This growth presents with broad pushing borders and has a good prognosis with less chances of local recurrence. However it can grow to large size, with invasion into adjacent bone and underlying structures. Surgery is the treatment of choice with a good margin clearance. Surgeons need to provide a full thickness tumor and uninvolved mucosa for an accurate diagnosis. ${ }^{4}$ Verrucous carcinoma of the genitor anal region is also called giant condyloma acuminatum of Buschke and Lowenstein. It commonly occurs on the glans penis and foreskin, which presents as verrucous, papillomatous proliferation. A deep biopsy is a must for this diagnosis. On Histopathology, the tumor invades with broad strands, which are large bulbous downward proliferations that compress the collagen bundles and push them aside. However there is no nuclear atypia, individual cell keratisation or horn perls which is a characteristic feature of squamous cell carcinoma.

Tuberculosis verrucosa cutis (TBVC), is yet another verrucous lesion, due to the cutaneous infection by mycobacterium tuberculosis. Cutaneous tuberculosis ranges from lupus vulgaris and TBVC at one end of the spectrum to the other end showing scrofuloderma and tuberculosis cutis orifacialis, as the cell mediated immunity decreases. Other forms of cutaneous tuberculosis include lichen scrofulosorum, papulonecrotic tuberculid and erythema nodosum. TBVC presents in many morphologic forms like psoriasiform, keloidal, crusted, exudative, sporotrichoid, destructive, tumor like and exuberant granulomatous forms. ${ }^{5}$ Its presentation can be as a solitary lesion clinically or have a multifocal presentation. Clinically the verrucous lesion may mimic a squamous cell carcinoma. ${ }^{6}$ There may be associated systemic manifestations in the form of lymphadenitis, pulmonary tuberculosis, abdominal tuberculosis and tubercular arthirits. Microscopy of all these variants are characterised by a caseating necrotising granulomatous inflammation with epithelioid cells and langhan giant cells. In TBVC, there is hyperkeratosis and acanthosis with an acute inflammatory infiltrate beneath the epidermis. There may be an abscess in the upper dermis, with classic granulomas in the mid dermis. Acid fast bacilli are more abundant in this condition and the bacilli can be demonstrated in histologic sections. Other granulomatous lesions can cause diagnostic differentials, like sarcoidosis, mycosis, leishmaniasis, leprosy, syphilis, foreign body implantation reaction and wegeners granulomatosis. 
The linear verrucous epidermal nevi also clinically appears as verrucous plaques and papules in a linear pattern, seen following Blashkos lines. It's also referred to as the verrucous epidermal nevus. They are congenital malformations or hamartomas derived from the embryonic ectoderm. The clinical presentation may be unilateral or may be seen as an extensive bilateral involvement. Sometimes, the multiple papules fuse in a serpiginous pattern. The histopathological features give the appearance of a raised papillary lesion with prominent acanthosis, papillomatosis and parakeratosis. ${ }^{7}$

Verrucous hemanigioma also presents as a cauliflower like growth, which is a rare congential vascular proliferation that may be mistaken for angiokeratoma. This lesion mainly presents at birth or in early childhood and the commonest location is the lower extremities. The original lesions are bluish red in colour, but frequent complications brings about secondary changes on the surface like verrucous or warty texture. Underlying the acanthotic epidermis with papillomatosis, there is a capillary proliferation resembling a hemangioma. The hemangiomatous component is greatly composed of dilated capillaries and larger cavernous, endothelium lined blood filled spaces. Inflammatory cells, hemosiderin and fibrosis may be present in the upper dermis. These features closely resemble angiokeratoma but in this condition, the lesion is limited to the papillary dermis whereas in verrucous hemangioma the lesion also extends into the subcutaneous fat. Immunohistochemical staining with endothelial markers like CD 31, CD 34 and GLUT 1 may highlight the endothelial cells, but the diagnosis can be made by light microscopic features. These markers are hepful in identifying the vascular origin of the tumor. They are a great help in cases where there are no lumina, to clearly recognise the lesion as a tumor of vascular differentiation.

It is very important to have a complete excision of the lesion, in order to study the morphology as well as to comment on the margin status. All the classic histopathological features may not be there in a small biopsy, hence a total excision with adequate margin clearance is a must. Thus the biopsy report will help the clinician to appropriately treat the lesion and it also helps to alleviate the anxieties of the patient. Simple excision is adequate in most of the cases, but in some lesions like verrucous carcinoma an adequate margin clearance is very important.

\section{References}

1. Rashmi GS Phulari, Khushbu Buddhdev, Rajendrasinh Rathore, Sweety Patel. Seborrheic keratosis J oral Maxillofac Pathol 2014, 18:32730.

2. Yu-Hung Wu, Pa-Fan Hsaio, Chi-Kuan Chen. Histopathologic and immunohistochemical distinction of condyloma and seborrheic keratosis in the genitofemoral area. De rmatologica Sinica.2013, 31(2):54-8.

3. Medina JE, Dichtel W, Luna MA. Verrucous squamous carcinoma of the oral cavity. A clinicopathologic study of 104 cases. Arch Oto Larngol.1984;110:437-40.

4. Mc Donald JS, Crissman JD, Gluckman JL. Verrucous carcinoma of the oral cavity. Head neck Surgery.1982;5:22-8.

5. Iizawa $\mathrm{O}$, Aiba $\mathrm{S}$, Tagami H. Tuberculosis verrucosa cutis ina tumor like form. $\mathrm{Br} \mathbf{J}$ Dermatol.1991;125:79-80.

6. Vora RV, Diwan NG, rathod KJ. Tuberculosis verrucosa cutis with multifocal involvement. Indian dermatol. Online J.2016;7:60-2.

7. Gorlin RJ, Lohen MM, Stephen LL. In Syndrome of the Head and neck, $3^{\text {rd }}$ Ed. New York; Oxford University Press;1990(Epidermal Nevus syndrome)pp362-6. 